\title{
4-DIMENSIONAL ZERO-HOPF BIFURCATION FOR POLYNOMIAL DIFFERENTIALS SYSTEMS WITH CUBIC HOMOGENEOUS NONLINEARITIES VIA AVERAGING THEORY
}

\author{
AMINA FEDDAOUI ${ }^{1}$, JAUME LLIBRE ${ }^{2}$ AND AMAR MAKHLOUF ${ }^{1}$
}

\begin{abstract}
The averaging theory of second order shows that for polynomial differential systems in $\mathbb{R}^{4}$ with cubic homogeneous nonlinearities at least nine limit cycles can be born in a zero-Hopf bifurcation.
\end{abstract}

\section{Introduction AND STATEMENT OF THE MAIN RESUlt}

Our goal is to study the periodic solutions which can bifurcate at a zero-Hopf bifurcation in a polynomial differential systems in $\mathbb{R}^{4}$ with cubic homogeneous nonlinearities by using the averaging theory of the second order.

In [7] the authors studied the zero-Hopf bifurcation in dimension $n>2$, by using the first order averaging method. They proved that at least $2^{n-3}$ limit cycles can bifurcate from one singularity with eigenvalues $\pm b i$ and $n-2$ zeros.

In [5] (resp. [2]) the authors studied the zero-Hopf bifurcation in polynomial differential systems in $\mathbb{R}^{3}$ (resp. $\mathbb{R}^{4}$ ) with quadratic homogeneous nonlinearities. By applying the averaging theory of the second order to these systems, they show that at most 3 limit cycles can bifurcate from a singular point having eigenvalues of the form $\pm b i$ and one zero (resp. two zeros). The zero-Hopf bifurcation in polynomial differential systems in $\mathbb{R}^{3}$ with cubic homogeneous nonlinearities has been studied recently in [3].

In this paper we are interested on the existence of periodic solutions bifurcating from the origin of coordinates of a polynomial differential systems in $\mathbb{R}^{4}$ with cubic homogeneous nonlinearities having eigenvalues $\pm b i$ and two zeros, i.e for the differential systems

$$
\begin{aligned}
& \dot{x}=\left(a_{1} \varepsilon+a_{2} \varepsilon^{2}\right) x-\left(b+b_{1} \varepsilon+b_{2} \varepsilon^{2}\right) y+\sum_{j=0}^{2} \varepsilon^{j} X_{j}(x, y, z, w), \\
& \dot{y}=\left(b+b_{1} \varepsilon+b_{2} \varepsilon^{2}\right) x+\left(a_{1} \varepsilon+a_{2} \varepsilon^{2}\right) y+\sum_{j=0}^{2} \varepsilon^{j} Y_{j}(x, y, z, w),
\end{aligned}
$$

2010 Mathematics Subject Classification. Primary: 34C07, 34C05, 34C40.

Key words and phrases. Hopf bifurcation, averaging theory, cubic polynomial differential systems. 


$$
\begin{aligned}
& \dot{z}=\left(c_{1} \varepsilon+c_{2} \varepsilon^{2}\right) z+\sum_{j=0}^{2} \varepsilon^{j} Z_{j}(x, y, z, w), \\
& \dot{w}=\left(d_{1} \varepsilon+d_{2} \varepsilon^{2}\right) w+\sum_{j=0}^{2} \varepsilon^{j} W_{j}(x, y, z, w),
\end{aligned}
$$

where

$$
\begin{aligned}
X_{j}(x, y, z, w)= & a_{j 0} x^{3}+a_{j 1} x^{2} y+a_{j 2} x^{2} z+a_{j 3} x^{2} w+a_{j 4} x y^{2}+a_{j 5} x y z+a_{j 6} x y w \\
& +a_{j 7} x z^{2}+a_{j 8} x z w+a_{j 9} x w^{2}+a_{j 10} y^{3}+a_{j 11} y^{2} z+a_{j 12} y^{2} w+a_{j 13} \\
& y z^{2}+a_{j 14} y z w+a_{j 15} y w^{2}+a_{j 16} z^{3}+a_{j 17} z^{2} w+a_{j 18} z w^{2}+a_{j 19} w^{3},
\end{aligned}
$$

$Y_{j}(x, y, z, w), Z_{j}(x, y, z, w)$ and $W_{j}(x, y, z, w)$ have the same expression as $X_{j}(x, y, z, w)$ by replacing $a_{j i}$ by $b_{j i}, c_{j i}$ and $d_{j i}$ for $j=0,1,2$ and $i=0,1, \ldots, 19$, respectively. The coefficients $a_{i j}, b_{i j}, c_{i j}, d_{i j}, a_{1}, a_{2}, b, b_{1}, b_{2}, c_{1}, c_{2}, d_{1}, d_{2}$ are real parameters with $b \neq 0$. Note that system (1) for $\varepsilon=0$ at the origin has eigenvalues $\pm b i, 0,0$. So for $\varepsilon=0$ the origin is a zero-Hopf equilibrium.

Our main result is the following one.

Theorem 1. By applying averaging theory of second order system (1) can exhibit at least 9 periodic solutions bifurcating from the origin when $\varepsilon=0$, and this number of periodic solutions is reached if and only if the following condition is satisfied $\left(3 a_{00}+\right.$ $\left.a_{04}+b_{01}+3 b_{010}\right) b \neq 0$.

Theorem 1 is proved in section 3. In section 2 we recall the averaging theory of first and second order as it was stated in [1]. This will be the main tool for proving Theorem 1.

\section{The AVERAging TheOrY of FIRSt AND SECOND ORDER}

The aim of this section is to present the averaging theory of first and second order as it was developed in $[1,4,6]$. The following result is Theorem 4.2 of [1].

Theorem 2. We consider the differential system

$$
\dot{x}(t)=\varepsilon F_{1}(t, x)+\varepsilon^{2} F_{2}(t, x)+\varepsilon^{3} R(t, x, \varepsilon),
$$

where $F_{1}, F_{2}: \mathbb{R} \times D \rightarrow \mathbb{R}^{n}, R: \mathbb{R} \times D \times\left(-\varepsilon_{f}, \varepsilon_{f}\right) \rightarrow \mathbb{R}^{n}$ are continuous functions, $T$-periodic in the first variable, and $D$ is an open subset of $\mathbb{R}^{n}$. Assume that the following hypotheses $(i)$ and $(i i)$ hold. Assume:

(i) $F_{1}, F_{2}, R$ are locally Lipschitz with respect to $x, F_{1}(t,.) \in C^{1}(D)$ for all $t \in \mathbb{R}$, and $R$ is differentiable with respect to $\varepsilon$. We define the averaging functions of 
first and second order $f_{1}, f_{2}: D \longrightarrow \mathbb{R}^{n}$ as

$$
\begin{aligned}
f_{1}(z) & =\frac{1}{T} \int_{0}^{T} F_{1}(s, z) d s \\
f_{2}(z) & =\frac{1}{T} \int_{0}^{T}\left[D_{z} F_{1}(s, z) \int_{0}^{s} F_{1}(t, z) d t+F_{2}(s, z)\right] d s .
\end{aligned}
$$

(ii) For $V \subset D$ an open and bounded set and for each $\varepsilon \in\left(-\varepsilon_{f}, \varepsilon_{f}\right) \backslash\{0\}$, there exists $a \in V$ such that $f_{1}(a)+\varepsilon f_{2}(a)=0$ and $d_{B}\left(f_{1}+\varepsilon f_{2}, V, a\right) \neq 0$.

Then for $|\varepsilon|>0$ sufficiently small there exists a T-periodic solution $x(t, \varepsilon)$ of the system (2) such that $x(0, \varepsilon) \rightarrow$ a when $\varepsilon \rightarrow 0$.

Where $d_{B}\left(f_{1}+\varepsilon f_{2}, V, 0\right)$ denotes the Brouwer degree of the function $f_{1}+\varepsilon f_{2}$ in the neighborhood $V$ of zero. It is known that if the function $f_{1}+\varepsilon f_{2}$ is $C^{1}$ then it is sufficient to check that $\operatorname{det}\left(D\left(f_{1}+\varepsilon f_{2}\left(a_{\varepsilon}\right)\right)\right) \neq 0$ in order to have that $d_{B}\left(f_{1}+\right.$ $\left.\varepsilon f_{2}, V, 0\right) \neq 0$, for more details see [8].

On the other hand if one of the real parts of the eigenvalues of the Jacobian matrix $D\left(f_{1}+\varepsilon f_{2}\right)\left(a_{\varepsilon}\right)$ is positive the periodic solution $x(t ; \varepsilon)$ is unstable. If all the real parts of the eigenvalues of this matrix are negative the periodic solution is locally stable. For a proof see Theorem 11.6 of [10].

For a general information on the averaging theory see for instance the books $[9,10]$.

\section{Proof of Theorem 1}

First we scale the variables $(x, y, z, w)$ doing the change of variables $(x, y, z, w)=$ $(\varepsilon X, \varepsilon Y, \varepsilon Z, \varepsilon W)$, second we pass to cylindrical coordinates doing $(X, Y, Z, W)=$ $(\rho \cos \theta, \rho \sin \theta, \eta, \xi)$, and third we take the angle $\theta$ as the new independent variable. Thus in the variables $(\rho, \eta, \xi)$ system (1) writes

$$
\begin{aligned}
& \frac{d \rho}{d \theta}=\varepsilon F_{11}(\theta, \rho, \eta, \xi)+\varepsilon^{2} F_{21}(\theta, \rho, \eta, \xi)+O\left(\varepsilon^{3}\right), \\
& \frac{d \eta}{d \theta}=\varepsilon F_{12}(\theta, \rho, \eta, \xi)+\varepsilon^{2} F_{22}(\theta, \rho, \eta, \xi)+O\left(\varepsilon^{3}\right), \\
& \frac{d \xi}{d \theta}=\varepsilon F_{13}(\theta, \rho, \eta, \xi)+\varepsilon^{2} F_{23}(\theta, \rho, \eta, \xi)+O\left(\varepsilon^{3}\right) .
\end{aligned}
$$


where

$$
\begin{aligned}
& F_{11}(\theta, \rho, \eta, \xi)=\frac{a_{1} \rho}{b}, \\
& F_{12}(\theta, \rho, \eta, \xi)=\frac{c_{1} \eta}{b} \\
& F_{13}(\theta, \rho, \eta, \xi)=\frac{d_{1} \xi}{b}, \\
& F_{21}(\theta, \rho, \eta, \xi)=\frac{1}{b^{2}}\left(-b_{1} \cos (\theta)^{2}-b_{1} \sin (\theta)^{2}\right)\left(a_{1} \rho \cos (\theta)^{2}+a_{1} \rho \sin (\theta)^{2}\right)+\frac{1}{b}\left(\left(a_{016} \eta^{3}+\right.\right. \\
& \left.a_{017} \eta^{2} \xi+a_{018} \eta \xi^{2}+a_{019} \xi^{3}\right) \cos (\theta)+\left(a_{2} \rho+a_{07} \eta^{2} \rho+a_{08} \eta \xi \rho+a_{09} \xi^{2} \rho\right. \\
& ) \cos (\theta)^{2}+\left(a_{02} \eta \rho^{2}+a_{03} \xi \rho^{2}\right) \cos (\theta)^{3}+a_{00} \rho^{3} \cos (\theta)^{4}+\left(b_{016} \eta^{3}+b_{017}\right. \\
& \left.\eta^{2} \xi+b_{018} \eta \xi^{2}+b_{019} \xi^{3}\right) \sin (\theta)+\left(\left(a_{013}+b_{07}\right) \eta^{2} \rho+\left(a_{014}+b_{08}\right) \eta \xi \rho+(\right. \\
& \left.\left.a_{015}+b_{09}\right) \xi^{2} \rho\right) \cos (\theta) \sin (\theta)+\left(\left(a_{05}+b_{02}\right) \eta \rho^{2}+\left(a_{06}+b_{03}\right) \xi \rho^{2}\right) \cos (\theta)^{2} \\
& \sin (\theta)+\left(a_{01}+b_{00}\right) \rho^{3} \cos (\theta)^{3} \sin (\theta)+\left(a_{2} \rho+b_{013} \eta^{2} \rho+b_{014} \eta \xi \rho+b_{015}\right. \\
& \left.\xi^{2} \rho\right) \sin (\theta)^{2}+\left(\left(a_{011}+b_{05}\right) \eta \rho^{2}+\left(a_{012}+b_{06}\right) \xi \rho^{2}\right) \cos (\theta) \sin (\theta)^{2}+\left(a_{04}\right. \\
& \left.+b_{01}\right) \rho^{3} \cos (\theta)^{2} \sin (\theta)^{2}+\left(b_{011} \eta \rho^{2}+b_{012} \xi \rho^{2}\right) \sin (\theta)^{3}+\left(a_{010}+b_{04}\right) \rho^{3} \\
& \left.\cos (\theta) \sin (\theta)^{3}+b_{010} \rho^{3} \sin (\theta)^{4}\right) \text {, } \\
& F_{22}(\theta, \rho, \eta, \xi)=\frac{1}{b^{2}}\left(b c_{2} \eta+b c_{016} \eta^{3}+b c_{017} \eta^{2} \xi+b c_{018} \eta \xi^{2}+b c_{019} \xi^{3}+b\left(c_{07} \eta^{2} \rho+c_{08} \eta \xi \rho\right.\right. \\
& \left.+c_{09} \xi^{2} \rho\right) \cos (\theta)+\left(-b_{1} c_{1} \eta+b c_{02} \eta \rho^{2}+b c_{03} \xi \rho^{2}\right) \cos (\theta)^{2}+b\left(c_{013} \eta^{2} \rho+\right. \\
& \left.c_{014} \eta \xi \rho+c_{015} \xi^{2} \rho\right) \sin (\theta)+b c_{00} \rho^{3} \cos (\theta)^{3}+b c_{01} \rho^{3} \cos (\theta)^{2} \sin (\theta)+b( \\
& \left.c_{05} \eta \rho^{2}+c_{06} \xi \rho^{2}\right) \sin (\theta) \cos (\theta)+\left(-b_{1} c_{1} \eta+b c_{011} \eta \rho^{2}+b c_{012} \xi \rho^{2}\right) \sin (\theta)^{2} \\
& \left.+b c_{04} \rho^{3} \cos (\theta) \sin (\theta)^{2}+b c_{010} \rho^{3} \sin (\theta)^{3}\right) \text {, } \\
& F_{23}(\theta, \rho, \eta, \xi)=\frac{1}{b^{2}}\left(b d_{2} \eta+b d_{016} \eta^{3}+b d_{017} \eta^{2} \xi+b d_{018} \eta \xi^{2}+b d_{019} \xi^{3}+b\left(d_{07} \eta^{2} \rho+d_{08} \eta \xi \rho\right.\right. \\
& \left.+d_{09} \xi^{2} \rho\right) \cos (\theta)+\left(-b_{1} d_{1} \eta+b d_{02} \eta \rho^{2}+b d_{03} \xi \rho^{2}\right) \cos (\theta)^{2}+b\left(d_{013} \eta^{2} \rho+\right. \\
& \left.d_{014} \eta \xi \rho+d_{015} \xi^{2} \rho\right) \sin (\theta)+b d_{00} \rho^{3} \cos (\theta)^{3}+b d_{01} \rho^{3} \cos (\theta)^{2} \sin (\theta)+b( \\
& \left.d_{05} \eta \rho^{2}+d_{06} \xi \rho^{2}\right) \sin (\theta) \cos (\theta)+\left(-b_{1} d_{1} \eta+b d_{011} \eta \rho^{2}+b d_{012} \xi \rho^{2}\right) \sin (\theta)^{2} \\
& \left.+b d_{04} \rho^{3} \cos (\theta) \sin (\theta)^{2}+b d_{010} \rho^{3} \sin (\theta)^{3}\right) \text {. }
\end{aligned}
$$

System (4) is written into the normal form (2) for applying the averaging theory taking

$$
\begin{aligned}
x=z & =(\rho, \eta, \xi), \\
t & =\theta \\
F_{1}(t, x) & =\left(F_{11}(\theta, \rho, \eta, \xi), F_{12}(\theta, \rho, \eta, \xi), F_{13}(\theta, \rho, \eta, \xi)\right), \\
F_{2}(t, x) & =\left(F_{21}(\theta, \rho, \eta, \xi), F_{22}(\theta, \rho, \eta, \xi), F_{23}(\theta, \rho, \eta, \xi)\right), \\
T & =2 \pi .
\end{aligned}
$$


From (3) we have that the first averaging function $f_{1}=\left(f_{11}, f_{12}, f_{13}\right)$ is

$$
f_{1 i}(\rho, \eta, \xi)=\frac{1}{2 \pi} \int_{0}^{2 \pi} F_{1 i}(\theta, \rho, \eta, \xi) d \theta .
$$

Doing these computations we get that

$$
f_{11}(\rho, \eta, \xi)=\frac{a_{1} \rho}{b}, \quad f_{12}(\rho, \eta, \xi)=\frac{c_{1} \eta}{b}, \quad f_{13}(\rho, \eta, \xi)=\frac{d_{1} \xi}{b} .
$$

Since we look for solutions $\left(\rho^{*}, \eta^{*}, \xi^{*}\right)$ of $f_{1}(\rho, \eta, \xi)=0$ with $\rho^{*}>0$, if $a_{1} \neq 0$ the first averaging function does not provide any information on the periodic solutions of the differential system (3). In order that the second averaging function can give information on the periodic solutions of the differential system (3) the first averaging function must be identically zero. So we take $a_{1}=c_{1}=d_{1}=0$, and compute the second averaging function.

Then from (3) we have that $f_{2}=\left(f_{21}, f_{22}, f_{23}\right)=\left(f_{21}(\rho, \eta, \xi), f_{22}(\rho, \eta, \xi), f_{23}(\rho, \eta, \xi)\right.$ is given by

$$
\begin{aligned}
f_{21}= & \frac{\rho}{8 b}\left(4\left(a_{07}+b_{013}\right) \eta^{2}+4\left(a_{08}+b_{014}\right) \eta \xi+\left(3 a_{00}+a_{04}+b_{01}+3 b_{010}\right) \rho^{2}+\right. \\
& \left.4\left(a_{09}+b_{015}\right) \xi^{2}+8 a_{2}\right), \\
(5) f_{22}= & \frac{1}{2 b}\left(2 c_{016} \eta^{3}+2 c_{2} \eta+2 \xi\left(c_{017} \eta^{2}+c_{018} \eta \xi+c_{019} \xi^{2}\right)+\left(c_{02}+c_{011}\right) \eta \rho^{2}+\right. \\
& \left.\left(c_{03}+c_{012}\right) \rho^{2} \xi\right), \\
f_{23}= & \frac{1}{2 b}\left(2 d_{016} \eta^{3}+2 d_{2} \eta+2 \xi\left(d_{017} \eta^{2}+d_{018} \eta \xi+d_{019} \xi^{2}\right)+\left(d_{02}+d_{011}\right) \eta \rho^{2}+\right. \\
& \left.\left(d_{03}+d_{012}\right) \rho^{2} \xi\right) .
\end{aligned}
$$

We isolate $\rho^{2}$ from the equation $f_{21}(\rho, \eta, \xi)=0$, and we substitute it in $f_{2 i}(\rho, \eta, \xi)=$ 0 for $i=2,3$. Then we get two polynomials $\left(g_{22}, g_{23}\right)=\left(g_{22}(\eta, \xi), g_{23}(\eta, \xi)\right)$ given by

$$
\begin{aligned}
& g_{22}=\frac{1}{\left(3 a_{00}+a_{04}+b_{01}+3 b_{010}\right) b}\left(C_{1} \eta+C_{2} \xi+C_{3} \eta \xi^{2}+C_{4} \eta^{2} \xi+C_{5} \eta^{3}+C_{6} \xi^{3}\right)=0, \\
& g_{23}=\frac{1}{\left(3 a_{00}+a_{04}+b_{01}+3 b_{010}\right) b}\left(D_{1} \eta+D_{2} \xi+D_{3} \eta \xi^{2}+D_{4} \eta^{2} \xi+D_{5} \eta^{3}+D_{6} \xi^{3}\right)=0,
\end{aligned}
$$

where

$$
\begin{aligned}
C_{1}= & -4 a_{2} c_{02}-4 a_{2} c_{011}+3 a_{00} c_{2}+a_{04} c_{2}+b_{01} c_{2}+3 b_{010} c_{2}, \\
C_{2}= & -4 a_{2}\left(c_{03}+c_{012}\right), \\
C_{3}= & -2\left(a_{08} c_{03}+a_{08} c_{012}+a_{09} c_{02}+a_{09} c_{011}+b_{014} c_{03}+b_{014} c_{012}+b_{015} c_{02}+b_{015} c_{011}\right)+ \\
& 3 a_{00} c_{018}+a_{04} c_{018}+b_{01} c_{018}+3 b_{010} c_{018}, \\
C_{4}= & -2\left(a_{07} c_{03}+a_{07} c_{012}+a_{08} c_{02}+a_{08} c_{011}+b_{013} c_{03}+b_{013} c_{012}+b_{014} c_{02}+b_{014} c_{011}\right)+ \\
& 3 a_{00} c_{017}+a_{04} c_{017}+b_{01} c_{017}+3 b_{010} c_{017}, \\
C_{5}= & -2\left(c_{02}+c_{011}\right)\left(a_{07}+b_{013}\right)+3 a_{00} c_{016}+a_{04} c_{016}+b_{01} c_{016}+3 b_{010} c_{016},
\end{aligned}
$$




$$
\begin{aligned}
C_{6}= & 3 a_{00} c_{019}+a_{04} c_{019}-2 a_{09} c_{03}-2 a_{09} c_{012}+b_{01} c_{019}+3 b_{010} c_{019}-2 b_{015} c_{03}-2 b_{015} c_{012}, \\
D_{1}= & -4 a_{2}\left(d_{02}+d_{011}\right)+3 a_{00} d_{2}+a_{04} d_{2}+b_{01} d_{2}+3 b_{010} d_{2}, \\
D_{2}= & -4 a_{2}\left(d_{03}+d_{012}\right), \\
D_{3}= & -2\left(a_{08} d_{03}+a_{08} d_{012}+a_{09} d_{02}+a_{09} d_{011}+b_{014} d_{03}+b_{014} d_{012}+b_{015} d_{02}+b_{015} d_{011}\right)+ \\
& 3 a_{00} d_{018}+a_{04} d_{018}+b_{01} d_{018}+3 b_{010} d_{018}, \\
D_{4}= & -2\left(a_{07} d_{03}+a_{07} d_{012}+a_{08} d_{02}+a_{08} d_{011}+b_{013} d_{03}+b_{013} d_{012}+b_{014} d_{02}+b_{014} d_{011}\right)+ \\
& 3 a_{00} d_{017}+a_{04} d_{017}+b_{01} d_{017}+3 b_{010} d_{017}, \\
D_{5}= & -2\left(d_{02}+d_{011}\right)\left(a_{07}+b_{013}\right)+3 a_{00} d_{016}+a_{04} d_{016}+b_{01} d_{016}+3 b_{010} d_{016}, \\
D_{6}= & 3 a_{00} d_{019}+a_{04} d_{019}-2 a_{09} d_{03}-2 a_{09} d_{012}+b_{01} d_{019}+3 b_{010} d_{019}-2 b_{015} d_{03}-2 b_{015} d_{012} .
\end{aligned}
$$

We suppose that $3 a_{00}+a_{04}+b_{01}+3 b_{010} \neq 0$.

Looking only at the coefficients of system (1) which appear in $C_{j}$ and $D_{j}$ we see that $C_{1}, C_{2}, C_{3}, C_{4}, C_{5}, C_{6}, D_{1}, D_{2}, D_{3}, D_{4}, D_{5}, D_{6}$ are all independent because the rank of the Jacobian matrix of the functions $C_{1}, C_{2}, C_{3}, C_{4}, C_{5}, C_{6}, D_{1}, D_{2}, D_{3}$, $D_{4}, D_{5}, D_{6}$ with respect to the variables $a_{00}, a_{04}, a_{07}, a_{08}, a_{09}, a_{2}, b_{01}, b_{010}, b_{013}, b_{014}$, $b_{015}, c_{02}, c_{03}, c_{011}, c_{012}, c_{016}, c_{017}, c_{018}, c_{019}, c_{2}, d_{02}, d_{03}, d_{011}, d_{012}, d_{016}, d_{017}, d_{018}, d_{019}$, $d_{2}$ is 12 , as it can be easily checked using maple or mathematica.

In short, since all coefficients of the polynomials $g_{22}(\eta, \xi)=0$ and $g_{23}(\eta, \xi)=0$ are independent they can be chosen arbitrary. By Bezout Theorem, we know that at most system $g_{22}(\eta, \xi)=0, g_{23}(\eta, \xi)=0$ has 9 solutions. We give an example of polynomial differential system with nine limit cycles

$$
\begin{aligned}
\dot{x} & =3 w^{2} x+x z^{2}+a_{013} y z^{2}+a_{016} z^{3}+\frac{x \varepsilon^{2}}{2}-y, \\
\dot{y} & =4 w^{2} y+(-4) x^{2} y+2 y z^{2}+\frac{y \varepsilon^{2}}{2}+x, \\
\dot{z} & =-4 w^{3}+w y^{2}+\frac{21 w^{2} z}{4}-\frac{3 x^{2} z}{2}-\frac{3 w z^{2}}{2}+2 z^{3}+z \varepsilon^{2}, \\
\dot{w} & =-7 w^{2} z+x^{2} z+y^{2} z-\frac{3 w z^{2}}{4}-\frac{15 z^{3}}{4}-\frac{z \varepsilon^{2}}{4} .
\end{aligned}
$$

From system (5) we have for system (6) that

$$
\begin{aligned}
f_{21}(\rho, \eta, \xi) & =\frac{1}{2} \rho\left(1+3 \eta^{2}+7 \xi^{2}-\rho^{2}\right) \\
f_{22}(\rho, \eta, \xi) & =\frac{1}{4}\left(8 \eta^{3}-6 \eta^{2} \xi+\eta\left(4+21 \xi^{2}-3 \rho^{2}\right)+2 \xi\left(-8 \xi^{2}+\rho^{2}\right)\right) \\
f_{23}(\rho, \eta, \xi) & =-\frac{1}{4} \eta\left(1+15 \eta^{2}+3 \eta \xi+28 \xi^{2}-4 \rho^{2}\right) .
\end{aligned}
$$


Solving system $(7)$ there are nine solutions $z_{i}=\left(\rho_{i}^{*}, \eta_{i}^{* *}, \xi_{i}^{*}\right)$ with $\rho_{i}^{*}>0$ for $i=$ $1, \ldots, 9$ given by

$$
\begin{aligned}
z_{1} & =(2,-1,0) \\
z_{2} & =(2 \sqrt{2}, 0,-1) \\
z_{3} & =(1,0,0) \\
z_{4} & =(2 \sqrt{2}, 0,1) \\
z_{5} & =(2,1,0) \\
z_{6} & =\left(\sqrt{\frac{1}{2}(55-13 \sqrt{7})},-\sqrt{3-\sqrt{7}}, \frac{1}{2}\left(-4 \sqrt{3-\sqrt{7}}+(3-\sqrt{7})^{\frac{3}{2}}\right)\right) \\
z_{7} & =\left(\sqrt{\frac{1}{2}(55-13 \sqrt{7})}, \sqrt{3-\sqrt{7}}, \frac{1}{2}\left(4 \sqrt{3-\sqrt{7}}-(3-\sqrt{7})^{\frac{3}{2}}\right)\right) \\
z_{8} & =\left(\sqrt{\frac{1}{2}(55+13 \sqrt{7})},-\sqrt{3+\sqrt{7}}, \frac{1}{2}\left(-4 \sqrt{3+\sqrt{7}}+(3+\sqrt{7})^{\frac{3}{2}}\right)\right) \\
z_{9} & =\left(\sqrt{\frac{1}{2}(55+13 \sqrt{7})}, \sqrt{3+\sqrt{7}}, \frac{1}{2}\left(4 \sqrt{3+\sqrt{7}}-(3+\sqrt{7})^{\frac{3}{2}}\right)\right) .
\end{aligned}
$$

Since the determinant

$$
\operatorname{det}\left(\left.\frac{\partial\left(f_{21}, f_{22}, f_{23}\right)}{\partial(\rho, \eta, \xi)}\right|_{(\rho, \eta, \xi)=\left(\rho^{*}, \eta^{*}, \xi^{*}\right)}\right)
$$

for these nine solutions $z_{i}=\left(\rho_{i}^{*}, \eta_{i}^{*}, \xi_{i}^{*}\right)$ are $-\frac{9}{2},-6, \frac{3}{8},-6,-\frac{9}{2},-\frac{9}{8}(-189+67 \sqrt{7})$, $-\frac{9}{8},(-189+67 \sqrt{7}), \frac{9}{8}(189+67 \sqrt{7}), \frac{9}{8}(189+67 \sqrt{7})$ respectively, we obtain, using the averaging theory of second order (see Theorem 2), 9 periodic solutions $\left(\rho_{i}(\theta, \varepsilon), \eta_{i}(\theta, \varepsilon), \xi_{i}(\theta, \varepsilon)\right)$ of system (4) such that $\left(\rho_{i}(0, \varepsilon), \eta_{i}(0, \varepsilon), \xi_{i}(0, \varepsilon)\right)=z_{i}$. In fact, these periodic soutions are limit cycles because the solutions $z_{i}$ are isolated solutions of system (7) because the determinants (8) are non-zero.

Going back through the changes of variables these 9 limit cycles provide 9 limit cycles $\left(x_{i}(t, \varepsilon), y_{i}(t, \varepsilon), z_{i}(t, \varepsilon), w_{i}(t, \varepsilon)\right.$ of the differential system $(1)$ such that

$$
\left(x_{i}(0, \varepsilon), y_{i}(0, \varepsilon), z_{i}(0, \varepsilon), w_{i}(0, \varepsilon)=\varepsilon\left(\rho_{i}^{*} \cos (b t), \rho_{i}^{*} \sin (b t), \eta_{i}^{*}, \xi_{i}^{*}\right)+O\left(\varepsilon^{2}\right) .\right.
$$

Since these initial conditions then to the origin of $\mathbb{R}^{4}$ when $\varepsilon \rightarrow 0$, the corresponding 9 limit cycles tend to the zero-Hopf singular point localized at the origin of $\mathbb{R}^{4}$. In short, the differential system (1) can exhibit a zero-Hopf bifurcation at the origin of system (6) with at least 9 limit cycles. This completes the proof of Theorem 1. 


\section{ACKNOWLEDGEMENTS}

This work is supported by the Ministerio de Economìa, Industria y competitividad, Agencia Estatal de Investigación grant MTM2016-77278-P (FEDER), the Agència de Gestió d'Ajusts Universitaris i de Recerca grant 2017SGR1617, and the European project Dynamics-H2020-MSCA-RISE-2017-777911.

\section{REFERENCES}

[1] A. Buică and J. Llibre, Averaging methods for finding periodic orbits via Brouwer degree, Bull. Sci. math. 128 (2004), 7-22.

[2] D. Djeddid, J. Llibre and A. Makhlouf, 4-dimensional zero-Hopf bifurcation via averaging theory, prepint, 2018.

[3] S. Kassa, J. Llibre and A. Makhlouf, 3-dimensional zero-Hopf ifurcation via averaging theory, preprint, 2018.

[4] J. Llibre, C.A. Buzzi and P.R. da Silva, 3-dimensional Hopf bifurcation via averaging theory, Discrete Contin. Dynam. Syst. 17 (2007), 529-540.

[5] J. Llibre, A. Makhlouf and S. Badi, 3-dimensional Hopf bifurcation via averaging theory of the second order. Discrete Contin. Dynam. Syst. 25(2009), 1287-1295.

[6] J. Llibre, A.C. Mereu and M.A. Teixeira, Limit cycles of the generalized polynomial Liénard differential equations, Math. Proc. Cambridge Philos. Soc. 148 (2010), 363-383.

[7] J. Llibre and X. Zhang, Hopf bifurcation in higher dimensional differential systems via the averaging method differential system in $\mathbb{R}^{d}$, Pacific J. Math. 240 (2009), 321-341.

[8] N.G. Lloyd, Degree theory, Cambridge Tracts in Mathematics 73,Cambridge University Press, Cambridge-New York-Melbourne, 1978.

[9] J.A. Sanders and F. Verhulst, Averaging methods in nonlinear dynamical systems, Applied Mathematical Sciences, New York, Springer-Verlag, 1985.

[10] F. Verhulst, Nonlinear differential equations and dynamical systems, Universitex, Berlin, Springer-Verlag, 1990.

1 Department of Mathematics, University of Annaba, Laboratory LMA, P.O.Box 12, Annaba 23000, Algeria

E-mail address: feddaouiamina90@gmail.com, makhloufamar@yahoo.fr

2 Departament de Matemàtiques, Universitat Autònoma de Barcelona, 08193 Bellaterra, Barcelona, Catalonia, Spain

E-mail address: jllibre@mat.uab.cat 